\title{
Revisionsvorschlag zum ambulanten Tarif abgelehnt
}

\author{
Jürg Schlup \\ Dr. med., Präsident der FMH
}

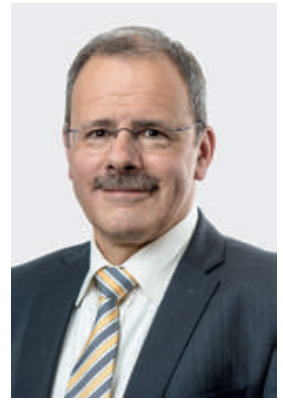

Mit dem Abschluss der Urabstimmung am 28. Mai 2016 zeigte sich schnell, dass lange und intensive Diskussionen unter Tarifpartnern und Ärztegesellschaften nicht zwangsläufig eine hohe Wahlbeteiligung begünstigen. Während des gut dreiwöchigen Abstimmungszeitraums machten nur 38\% unserer Mitglieder von ihrem Stimmrecht Gebrauch. Fast zwei von drei Mitgliedern reichte ihre knappe Freizeit nicht, um sich in ein komplexes Thema einzulesen und sich mit viel Aufwand eine fundierte Meinung zu bilden. Die weit auseinanderliegenden Abstimmungsempfehlungen der Ärzteorganisationen dürften die Beteiligung zusätzlich reduziert haben: Wer mit gegensätzlichen Aussagen verschiedener Ärztevertreter konfrontiert ist und die Fakten nicht im Detail beurteilen kann, wird seine Möglichkeit abzustimmen eher ungenutzt lassen.

Ein mit allen Ärzteorganisationen entwickelter und demokratisch abgestützter Tarifvorschlag ist für uns nach wie vor der richtige Weg.

Diejenigen, die abstimmten, haben ihre Meinung allerdings deutlich zum Ausdruck gebracht. Das Nein zum vorgelegten revidierten Tarif zeigt, dass die Ärzteschaft weder auf Dauer bereit ist, das Dogma der Kostenneutralität zu akzeptieren noch sich den knappen Zeitvorgaben des Bundesrats zu fügen. Diese Haltung ist nachvollziehbar und verständlich: Praxisambulant tätige Ärzte behandeln im Jahr 80\% der Bevölkerung - und verursachen lediglich 15\% der gesamten Gesundheitskosten. Und obwohl immer mehr Gesundheitsprobleme

Resultate der Urabstimmung zur Tarifrevision mit den Empfehlungen der Ärztekammer:

\begin{tabular}{lll}
\hline & $\begin{array}{l}\text { Resultate der } \\
\text { Urabstimmung } \\
\text { vom 9. Juni 2016 }\end{array}$ & $\begin{array}{l}\text { Empfehlung der } \\
\text { 28. April 2016 }\end{array}$ \\
\hline Tarifstruktur ats-tms Version 1.0 & NEIN (54\%) & JA (70\%) \\
\hline $\begin{array}{l}\text { Modellansatz «individueller ärztlicher Faktor» } \\
\text { IAF }\end{array}$ & NEIN (87\%) & NEIN (52\%) \\
\hline $\begin{array}{l}\text { Stichfrage: Falls IAF abgelehnt wird: } \\
\text { Tarifstruktur auch in diesem Fall annehmen? }\end{array}$ & NEIN (56\%) & JA (65\%) \\
\hline $\begin{array}{l}\text { Normierungsvereinbarung Version 0.3 vom } \\
\text { 25.3.2016 mit dem Normierungsfaktor 0,73 }\end{array}$ & NEIN (89\%) & NEIN (53\%) \\
\hline $\begin{array}{l}\text { Stichfrage: Falls Normierungsvereinbarung } \\
\text { abgelehnt: Annahme unter Voraussetzung Korrektur } \\
\text { innert 5-10 Jahren? }\end{array}$ & NEIN (60\%) & JA (56\%) \\
\hline $\begin{array}{l}\text { Grundvertrag KVG Version 10.2 vom 8.3.2016 } \\
\text { Grundvertrag UVG Version 0.7 vom 8.3.2016 }\end{array}$ & NEIN (55\%) & JA (70\%) \\
\hline
\end{tabular}

ambulant statt stationär behandelt werden, wachsen die Kosten des praxisambulanten Sektors nicht überproportional, sondern entwickeln sich seit Einführung des KVG 1996 vergleichbar mit denjenigen der übrigen Gesundheitsversorgung.

Weil der Tarif auf Daten aus den 90er Jahren basiert und seit 2004 weitgehend unverändert geblieben ist, prägt die Kostenneutralität schon lange den Praxisalltag. Dass ein betriebswirtschaftlich berechneter und sachgerecht revidierter Tarif um 27\% abgewertet werden müsste, um Kostensteigerungen zu vermeiden, ist letztlich das Resultat dieser langen Stagnation. Bedenkt man weiter, dass bei einer Umverteilung innerhalb eines normierten Tarifs zwangsläufig jedem Zugewinn ein gleich hoher Verlust gegenübersteht, wird deutlich, wie ehrgeizig das Werben um Akzeptanz hierfür war.

Wo die Vertreter der Ärzteorganisationen in der Ärztekammer mehrheitlich den Weg empfohlen haben, durch das fristgerechte Vorlegen eines normierten Tarifvorschlags am Verhandlungstisch zu bleiben, zeigt die Basis, dass sie diesen Platz zu riskieren bereit ist. Die Politik der letzten Jahre hat angesichts erfolgter und angedrohter Tarifeingriffe bei vielen Ärztinnen und Ärzten das Gefühl hinterlassen, dass die Tarifpartnerschaft keine ausreichende Handlungsfreiheit geniesst und sich lediglich vor der subsidiären Kompetenz des Bundesrats hertreiben lässt. Dem haben sich unsere Mitglieder nun verweigert.

Die in dieser Abstimmung demokratisch ermittelte Position der Ärzteschaft verstehen wir als Auftrag: Ein Tarif, der von der Mehrheit der Abstimmenden nicht akzeptiert wird, verlangt Nachbesserungen - unabhängig von den Zeitlimiten des Bundesrats. Die FMH wird darum keinen Tarif einreichen und die ärztlichen Gegner des Vorschlages für eine gemeinsame Entwicklung von Lösungen einladen. Ein unter Einbezug aller Ärzteorganisationen entwickelter und demokratisch abgestützter Tarifvorschlag ist für uns nach wie vor der richtige Weg. Vor allem wird die FMH aber alles daran setzen, die Verhandlungen weiterzuführen, und verdeutlichen, dass ein tragfähiger Arzttarif die Unterstützung der Ärzteschaft braucht. In diesem Sinne werden wir auf unsere Tarifpartner und die Genehmigungsbehörden zugehen. 